\title{
ERRATUM
}

\section{Erratum to: Trends in Chronologic Age and Infant Respiratory Syncytial Virus Hospitalization: an 8-Year Cohort Study}

Jon P. Fryzek · William J. Martone · Jessie R. Groothuis

() Springer Healthcare 2011

Erratum to: Fryzek JP, Martone WJ, Groothuis JR. Trends in chronologic age and infant respiratory syncytial virus hospitalization: an 8-year cohort study. Adv Ther. 2011;28(3):195-201.

The authors of the above-mentioned paper noticed a small error in one of the results that was reported. In the Abstract (page 195) and in the Discussion (page 200) section of the manuscript, it states that "the proportion of RSVH in the first year of life was lowest among infants aged $<1$ month (0.9\%)." The correct percent is $9 \%$. This percentage is correctly reported in the Results section and Figure 3 of the manuscript. This error does not impact any of the other results reported in the paper nor does it change the conclusions. The authors regret this error, and thank Springer Healthcare for publishing the correction. 